\title{
Principais fatores de risco relacionados ao desenvolvimento de diabetes gestacional
}

\author{
Main risk factors related to the development of gestational diabetes \\ Principales factores de riesgo relacionados con el desarrollo de la diabetes gestacional \\ Taiane Lima dos Santos ${ }^{1 *}$, Cleuson Vieira Costa ${ }^{2}$, Elisete Silva Amorim ${ }^{3}$, Edilene Bispo Gomes ${ }^{4}$, \\ Hadsan Taiana Aleixo da Fonseca ${ }^{5}$, Luiz Carlos Araújo de Souza ${ }^{6}$, Silvio Douglas Medeiros Costa ${ }^{5}$, \\ Simone Ribeiro Vieira7 , Silvia Maria dos Santos Sousa ${ }^{5}$, Aylia Virgínia Oliveira Cardosoํ.
}

\section{RESUMO}

Objetivo: Buscar na literatura científica evidencias acerca dos fatores de risco para desenvolver diabetes gestacional. Métodos: O presente estudo trata-se de uma revisão integrativa da literatura. Realizou-se a busca dos estudos nas bases de dados Literatura Latino Americana e do Caribe em Ciências da Saúde (LILACS) e a Biblioteca Virtual em Saúde (BVS). Para a busca dos estudos realizou-se a associação dos descritores "Diabetes gestacional", "Gravidez", "Hiperglicemia". Foram incluídos na busca os estudos primários, que estivessem disponíveis em sua totalidade, publicados nos últimos 5 anos, em qualquer idioma. Foram excluídos da busca inicial os resumos, capítulos de livros, teses de doutorado, textos incompletos, relatos técnicos e dissertações de mestrados. Resultados: Todos os estudos estavam no idioma português $(100 \%)$, a maioria foram publicados no ano de 2020 (30\%) e tratavam-se de estudos de campo (76\%). Com base nos achados, emergiram as seguintes categorias: Principais fatores de risco relacionados ao desenvolvimento de DMG, Complicações materno-fetais em gestantes com DMG, Diagnóstico e Tratamento. Considerações finais: Evidenciou-se que a obesidade, o excesso de peso e a péssima nutrição das gestantes estiveram maior relação ao desenvolvimento da DMG, assim como a hipertensão.

Palavras-chave: Diabetes gestacional, Gravidez, Hiperglicemia.

\begin{abstract}
Objective: Search the scientific literature for evidence about risk factors for developing gestational diabetes. Methods: This study is an integrative literature review. The search for studies was carried out in the Latin American and Caribbean Literature in Health Sciences (LILACS) and Virtual Health Library (VHL) databases. To search for the studies carried out, the association of the descriptors "Gestational diabetes", "Pregnancy", "Hyperglycemia" was used. Primary studies that were available in their entirety, published in the last 5 years, in any language, were included in the search. Abstracts, book chapters, doctoral theses, incomplete texts, technical reports and master's dissertations were excluded from the initial search. Results: All studies were in Portuguese (100\%), most were published in $2020(30 \%)$ and they were field studies $(76 \%)$. Based on the findings, the following categories emerged: Main risk factors related to the development of GDM, Maternal-
\end{abstract}

\footnotetext{
${ }^{1}$ Faculdade de Ciência e Tecnologia do Maranhão (FACEMA), Caxias - MA.

*E-mail: thaylimasantos@hotmail.com

2 Faculdade Estácio de Macapá, Belém - PA.

${ }^{3}$ Faculdade Metropolitana da Amazônia (FAMAZ), Belém - PA.

${ }^{4}$ Centro Universitário do Estado do Pará (CESUPA), Belém - PA.

5 Universidade da Amazônia (UNAMA), Belém - PA.

6 Centro Universitário Metropolitano da Amazônia, Belém - PA.

7 Centro Universitário de Lavras, Brasília - DF.
}

SUBMETIDO EM: 12/2021 
fetal complications in pregnant women with GDM, Diagnosis and Treatment. Final considerations: It was evident that obesity, overweight and poor nutrition in pregnant women were more related to the development of GDM, as well as hypertension.

Key words: Gestational diabetes, Pregnancy, Hyperglycemia.

\begin{abstract}
Objetivo: Buscar en la literatura científica evidencia sobre factores de riesgo para desarrollar diabetes gestacional. Métodos: Este estudio es una revisión integradora de la literatura. La búsqueda de estudios se realizó en las bases de datos de Literatura Latinoamericana y Caribeña en Ciencias de la Salud (LILACS) y Biblioteca Virtual en Salud (BVS). Para la búsqueda de los estudios realizados se utilizó la asociación de los descriptores "Diabetes gestacional", "Embarazo", "Hiperglucemia". Se incluyeron en la búsqueda los estudios primarios que estaban disponibles en su totalidad, publicados en los últimos 5 años, en cualquier idioma. Se excluyeron de la búsqueda inicial resúmenes, capítulos de libros, tesis doctorales, textos incompletos, informes técnicos y disertaciones de maestría. Resultados: Todos los estudios fueron en portugués (100\%), la mayoría fueron publicados en 2020 (30\%) y fueron estudios de campo (76\%). Con base en los hallazgos, surgieron las siguientes categorías: Principales factores de riesgo relacionados con el desarrollo de DMG, Complicaciones materno-fetales en mujeres embarazadas con DMG, Diagnóstico y tratamiento. Consideraciones finales: Se evidenció que la obesidad, el sobrepeso y la desnutrición en las embarazadas estaban más relacionados con el desarrollo de DMG, así como con la hipertensión.
\end{abstract}

Palabras clave: Diabetes gestacional. Embarazo, Hiperglucemia.

\title{
INTRODUÇÃO
}

A Diabetes Mellitus Gestacional (DMG) é uma condição clínica que acomete mulheres grávidas, no qual tem início e diagnóstico da diabetes ou intolerância à glicose durante o período gestacional, podendo persistir ou não após o parto (MENICATTI M, et al., 217). A prevalência da DMG a nível mundial varia entre $1 \%$ a $28 \%$, dependendo dos critérios de diagnósticos e características da população. No Brasil a prevalência da DMG está estimada em torno de 18\% no SUS (SANTOS WMS, et al., 2019).

De acordo com Batista MJ, et al. (2020), a DMG consiste em uma disfunção pancreática, que pode ser branda ou grave, que pode causar no indivíduo que é acometido desde um simples funcionamento inadequado até a perda total de sua capacidade de produção de insulina. Sendo esta uma síndrome clínica que causa hiperglicemia devido a deficiência na ef etividade da insulina e a sua fisiopatologia está relacionada ao aumento de hormônios contrarreguladores da insulina, causado pelo estresse fisiológico imposto pela gravidez, além de fatores predeterminantes genéticos e ambientais (FERREIRA AF, et al., 2018).

O principal hormônio relacionado com a redução da sensibilidade e resistência à insulina é o lactogênio placentário, contudo, sabe-se que outros hormônios hiperglicemiantes como cortisol, estrógeno, progesterona e prolactina também estão envolvidos (SOUSA AL, et al., 2017).

$\mathrm{Na}$ diabetes gestacional o bebê é exposto a grandes quantidades de glicose ainda no ambiente intrauterino fato este que interfere no desenvolvimento do embrião e causa maior risco de crescimento fetal excessivo (macrossomia fetal), assim como partos traumáticos, hipoglicemia neonatal e até obesidade e diabetes na vida adulta do bebê. Os estudos observaram o aumento de até três vezes com relação aos casos de malformações congênitas em gestante diabética, bem como o aumento de até dez vezes nos partos prétermo (QUEIROZ A, et al., (2018).

Além de ser uma das principais causas de morbimortalidade materna, e está associada a Síndromes Hipertensivas na Gestação (SHG) (hipertensão, pré-eclâmpsia e eclampsia), bem como no aumento das taxas de cesariana, macrossomia e hiperinsulinismo fetal. $E$ também à problemas a longo prazo como Diabetes Mellitus tipo 2 (DM2), síndrome metabólica e doença cardiovascular (MARIANO TF, et al., 2021). 
Dentre os principais fatores de risco da DMG destacam-se a idade avançada, história familiar de DM2 em parentes de primeiro grau, síndrome de ovários policísticos e baixa estatura, além do excesso de peso e ganho ponderal acima do recomendado (OLIVEIRA ACV, et al., 2021).

O pré-natal tem o objetivo assim como entre outras atribuições, identificar precocemente as alterações que acontecem durante a gestação que coloquem em risco a saúde da mulher e do feto, tendo está uma importante atuação na prevenção, evitando desta forma complicações pré, peri e pós-natais, bem como parto prematuro, aborto espontâneo, pré-eclâmpsia, DMG, entre outros fatores (SOUZA W, et al., 2021).

O rastreamento para DMG é realizado a partir da $24^{a}$ semana de gestação, por meio do exame de glicemia em jejum e o diagnóstico da DMG pode ser realizado por meio de testes provocativos com sobrecarga de glicose (Teste Oral de Tolerância Glicose (TOTG), medição de glicose no plasma (após jejum mínimo de 8 horas) que deve ser feito no segundo trimestre da gestação, bem como a triagem precoce de gestantes de alto risco na primeira consulta pré-natal (FERNANDES CN, et al., 2020).

Diante do exposto, o presente estudo teve o objetivo de buscar na literatura cientifica evidencias acerca dos fatores de risco para desenvolver diabetes gestacional.

\section{MÉTODOS}

O presente estudo trata-se de uma revisão integrativa da literatura, este tipo de estudo é realizado com base em práticas baseadas em evidências, resumindo o passado da literatura empírica ou teórica, com o intuito de fornecer maior compreensão quanto ao um fenômeno particular.

Para a elabora do estudo realizou-se algumas fases, tais como: definição do objetivo, determinação dos critérios de inclusão e exclusão, bem como a definição das informações a serem extraídas dos artigos selecionados, análise e a discussão dos resultados encontrados.

Houve a formulação da seguinte questão norteadora com o objetivo de orientar a elaboração do estudo: Quais evidências cientifica existente na literatura quanto aos fatores de risco para desenvolver diabetes gestacional?

Utilizou-se as bases de dados Literatura Latino Americana e do Caribe em Ciências da Saúde (LILACS) e a Biblioteca Virtual em Saúde (BVS). Foi realizado uma buscar na produção científica relacionada aos fatores associados ao desenvolvimento da diabetes gestacional, utilizando os seguintes descritores: "Diabetes gestacional”, "Gravidez", "Hiperglicemia".

Foi incluído na busca dos estudos as pesquisas que estavam disponíveis em sua totalidade, que foram publicados nos últimos 5 anos, nos idiomas que atendessem questão do estudo. Foram excluídos da busca os capítulos textos incompletos, livros, resumos, teses de doutorado, relatos técnicos. A pesquisa ocorreu em Dezembro de 2021.

Os estudos foram pré-selecionados segundo os critérios de inclusão e exclusão e de acordo com a estratégia de funcionamento e busca de cada base de dados, obtendo -se 162 estudos como busca geral na LILACS, sendo que limitando a busca para texto completo e para os últimos cinco anos obteve-se 30 estudos, destes foram analisados títulos e resumos e apenas 5 estudos foram foi condizente com a questão desta pesquisa.

Na base BVS, como busca total foram encontrados 105 estudos, aplicando na pesquisa o filtro que limita texto completo e nos anos de 2017, 2018, 2019, 2020 e 2021 obteve-se 15 estudos, destes foram analisados títulos e resumos onde apenas 8 estudos foi condizente com a questão desta pesquisa.

A segunda fase foi composta pela análise dos estudos quanto ao potencial de participação no estudo, analisando se atendiam a questão da pesquisa, assim como o tipo de investigação, amostra objetivos, desfechos, método, resultados e conclusão. A busca final resultou em 13 artigos que foram adicionados ao estudo, como mostra a Figura 1. 
Figura 1 - Fluxograma do processo de seleção dos estudos para a revisão integrativa.

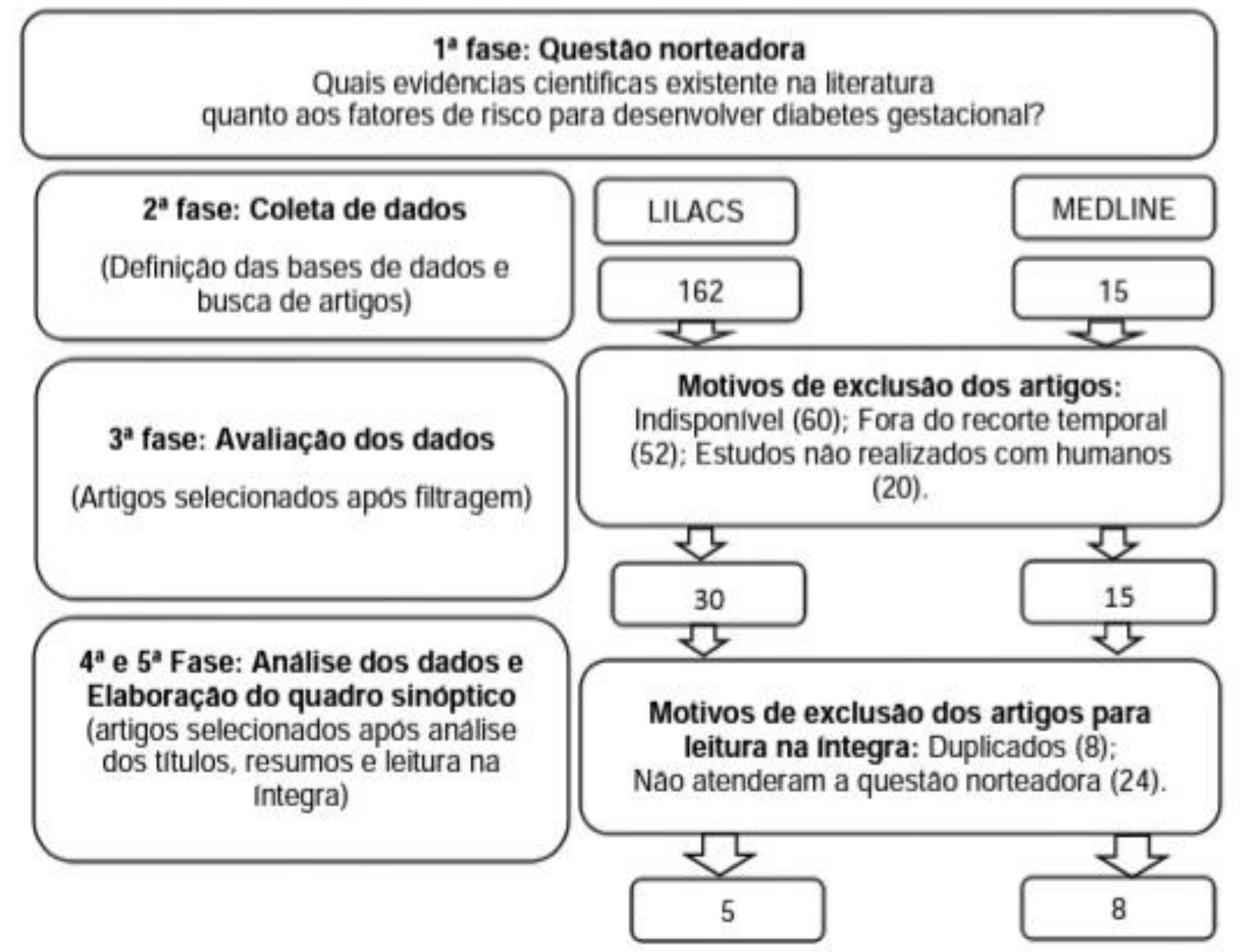

Fonte: Carvalho BL, et al., 2021.

\section{RESULTADOS E DISCUSSÃO}

A análise dos estudos possibilitou analisar os fatores associados ao desenvolvimento da diabetes gestacional, desta forma pode-se observar que todos os estudos estavam no idioma português (100\%), a maioria foram publicados no ano de 2020 (30\%) e tratavam-se de estudos de campo (76\%).

Os 13 estudos incluídos nesta revisão foram organizados segundo os autores e ano, método, objetivo e principais resultados (Quadro 1). 
Quadro 1 - Apresentação dos estudos segundo o autor, amostra, objetivo e principais resultados.

\begin{tabular}{|c|c|c|c|}
\hline Autor & Método & Objetivo & Resultado \\
\hline ARAÚJO IM, et al., 2020 & $\begin{array}{l}\text { Revisão da } \\
\text { literatura. }\end{array}$ & $\begin{array}{l}\text { Apresentar o risco da diabetes na gravidez e } \\
\text { destacar os cuidados do enf ermeiro às } \\
\text { pacientes. }\end{array}$ & $\begin{array}{l}\text { A DMG alcança cerca de } 25 \% \text { das mulheres grávidas no mundo. } \\
\text { Nota-se que o aumento dos níveis de glicose no sangue, trazem } \\
\text { complicações à saúde da mulher e da criança. }\end{array}$ \\
\hline GUERRA JVV, et al., 2019 & $\begin{array}{l}\text { Estudo de } \\
\text { campo. }\end{array}$ & $\begin{array}{c}\text { Analisar a assistência pré-natal a partir do } \\
\text { número de consultas obstétricas e nutricionais } \\
\text { na gestação e a relação com o diabetes } \\
\text { gestacional. }\end{array}$ & $\begin{array}{l}\text { Evidenciou-se a necessidade de melhoria quanto ao } \\
\text { atendimento da mulher no pré-natal de alto risco, em especial, } \\
\text { quando há lacunas no acesso aos serviços especializados. }\end{array}$ \\
\hline $\begin{array}{l}\text { KUNZENDORFF BA, et al., } \\
2017\end{array}$ & $\begin{array}{l}\text { Revisão da } \\
\text { literatura. }\end{array}$ & $\begin{array}{l}\text { Apresentar as possíveis causas do } \\
\text { desenvolvimento da diabetes gestacional, além } \\
\text { da fisiopatologia, prováveis complicações e } \\
\text { consequências no período pré e pós-parto. }\end{array}$ & $\begin{array}{l}\text { A diabetes gestacional é uma patologia comum e que há } \\
\text { diversos meios para prevenção e tratamento. Além disso, as } \\
\text { complicações materno fetais também são abordadas e } \\
\text { associadas às formas de se evitar tais problemas. }\end{array}$ \\
\hline MARTINS GKF, et al., 2020 & $\begin{array}{l}\text { Estudo de } \\
\text { campo. }\end{array}$ & $\begin{array}{l}\text { Estimar a prevalência e preditores de diabetes } \\
\text { mellitus gestacional (DMG) em um serviço de } \\
\text { alta complexidade. }\end{array}$ & $\begin{array}{l}\text { A prevalência de DMG foi de } 18,5 \% \text {. Quanto aos fatores de } \\
\text { risco, gestantes que tiveram glicemia em jejum alterada tiveram } \\
\text { uma probabilidade } 16 \% \text { maior de ter DMG. Alteração no teste } \\
\text { oral de tolerância à glicose (TOTG } 1 \mathrm{~h} \text { ) indicou } 6 \% \text { mais chances } \\
\text { para DMG do que aquelas sem alteração no TOTG } 1 \mathrm{~h} \text {. }\end{array}$ \\
\hline MORAIS AM, et a., 2018 & $\begin{array}{l}\text { Estudo de } \\
\text { campo. }\end{array}$ & $\begin{array}{l}\text { Avaliar o perfil epidemiológico, socioeconômico, } \\
\text { clínico-obstétrico e identificar o conhecimento } \\
\text { em relação ao DMG de gestantes atendidas em } \\
\text { um Centro Especializado de Saúde da Mulher } \\
\text { de uma Unidade Básica de Saúde (UBS) de } \\
\text { Lajeado/RS. }\end{array}$ & $\begin{array}{l}\text { Os resultados mostraram que as grávidas não possuíam } \\
\text { conhecimento sobre diabetes. A maioria }(90 \%) \text { não tinha doença } \\
\text { prévia à gestação, } 55 \% \text { utilizavam medicação durante a } \\
\text { gestação, } 65 \% \text { não praticava atividade física e } 65 \% \text { não } \\
\text { consultou nutricionista nos últimos } 12 \text { meses. Nenhuma das } \\
\text { gestantes possuía Diabetes Mellitus ou teve DMG em gestação } \\
\text { prévia, contudo uma participante (5\%) apresentava DMG. }\end{array}$ \\
\hline $\begin{array}{l}\text { ZUCCOLOTTO DCC, et al., } \\
2019\end{array}$ & $\begin{array}{l}\text { Estudo de } \\
\text { campo. }\end{array}$ & $\begin{array}{l}\text { Investigar a relação entre os padrões } \\
\text { alimentares de gestantes com o excesso de } \\
\text { peso materno e o diabetes mellitus gestacional. }\end{array}$ & $\begin{array}{l}\text { Observou-se que mulheres com maior adesão ao padrão } \\
\text { "saudável" (OR = 0,52; IC95\% 0,33-0,83) e "tradicional } \\
\text { brasileiro" (OR =0,61; IC95\% 0,38-0,96) apresentaram menor } \\
\text { chance de obesidade que as mulheres com menor adesão, } \\
\text { independentemente de fatores de conf usão. Após ajuste pelo } \\
\text { excesso de peso materno, não houve associação entre os } \\
\text { padrões alimentares e o diabetes mellitus gestacional. }\end{array}$ \\
\hline BORGES MCV, et al., 2017 & $\begin{array}{l}\text { Estudo de } \\
\text { campo. }\end{array}$ & $\begin{array}{l}\text { Analisar o conhecimento das gestantes sobre } \\
\text { Diabetes Mellitus Gestacional (DMG) e } \\
\text { identificar os fatores de riscos. }\end{array}$ & $\begin{array}{c}\text { Os resultados obtidos demonstraram que 88\% das gestantes } \\
\text { desconhecem sobre a DMG e suas implicações para o binômio } \\
\text { mãe/filho. }\end{array}$ \\
\hline
\end{tabular}




\begin{tabular}{|c|c|c|c|}
\hline Autor & Método & Objetivo & Resultado \\
\hline CYSNEIROS GF, et al., 2020 & $\begin{array}{c}\text { Estudo de } \\
\text { campo. }\end{array}$ & $\begin{array}{c}\text { Avaliar o estado nutricional e consumo alimentar } \\
\text { de gestantes diabéticas tipo } 2 \text { e diabetes } \\
\text { gestacional. }\end{array}$ & $\begin{array}{c}\text { A maior parte da amostra encontrava-se em excesso de peso. } \\
\text { As sedentárias demonstraram ingestão elevada em todos os } \\
\text { macronutrientes }(p>0,05) \text {. As internadas, maiores ingestões de } \\
\text { energia e demais macronutrientes }(p<0,001) \text { comparadas às } \\
\text { atendidas no ambulatório. }\end{array}$ \\
\hline FALEIROS GQA, et al., 2021 & $\begin{array}{l}\text { Estudo de } \\
\text { campo. }\end{array}$ & $\begin{array}{l}\text { Comparar o peso dos recém-nascidos de } \\
\text { gestantes diabéticas gestacionais e } \\
\text { metabolicamente normais. }\end{array}$ & $\begin{array}{l}\text { A comparação entre a média de peso do recém-nascido em } \\
\text { pacientes com diabetes em uso de insulina ( } 3287 \pm 350 \mathrm{~g}) \text { e não } \\
\text { usuárias de insulina ( } 3178 \pm 474 \mathrm{~g}) \text { que também não foi } \\
\text { significativa }(p=0,448) \text {. Não houve associação entre o peso } \\
\text { materno (peso inicial, peso final, ganho de peso) e peso do } \\
\text { recém-nascido, avaliados através da regressão linear }(p=0,448)\end{array}$ \\
\hline FRIEDRICH F, et al., 2019 & $\begin{array}{l}\text { Revisão da } \\
\text { literatura. }\end{array}$ & $\begin{array}{l}\text { Identificar os fatores que interferem na adesão } \\
\text { ao tratamento da DMG. }\end{array}$ & $\begin{array}{l}\text { A gestante portadora de DMG não tratada tem maior risco de } \\
\text { rotura prematura de membranas, parto pré-termo, feto com } \\
\text { apresentação pélvica e feto macrossômico, assim como o risco } \\
\text { elevado de pré-eclâmpsia. }\end{array}$ \\
\hline GUERRA JVV, et a., 2018 & $\begin{array}{c}\text { Estudo de } \\
\text { campo. }\end{array}$ & $\begin{array}{l}\text { Identificar o número de casos de diabetes } \\
\text { gestacional e correlacionar estado nutricional } \\
\text { pré-gestacional e diabetes mellitus gestacional } \\
\text { em mulheres atendidas no ambulatório de } \\
\text { Ginecologia e Obstetrícia de um Hospital } \\
\text { Universitário em Niterói. }\end{array}$ & $\begin{array}{c}\text { Cerca de } 14 \% \text { das gestantes }(n=25) \text { desenvolveram diabetes, } \\
24 \% \text { iniciaram a gestação com sobrepeso e } 29,5 \% \text {, com } \\
\text { obesidade. }\end{array}$ \\
\hline NERIS VA, et a., 2021 & $\begin{array}{l}\text { Estudo de } \\
\text { campo. }\end{array}$ & $\begin{array}{c}\text { Analisar o ganho ponderal e o estado nutricional } \\
\text { de gestantes portadoras de diabetes mellitus } \\
\text { gestacional. }\end{array}$ & $\begin{array}{c}\text { O ganho ponderal acima do recomendado foi predominante } \\
\text { (45,3\%), revelando associação positiva com o estado nutricional } \\
\text { atual. A presença de patologias associadas ao DMG foi a única } \\
\text { variável obstétrica, que apresentou associação significativa com } \\
\text { o estado nutricional prévio, atual e ganho ponderal gestacional, } \\
\text { independentemente do tipo de comorbidades. }\end{array}$ \\
\hline $\begin{array}{l}\text { NOGUEIRA MDA, et al., } \\
\qquad 2020\end{array}$ & $\begin{array}{l}\text { Estudo de } \\
\text { campo. }\end{array}$ & $\begin{array}{l}\text { Verificar existência de relação entre o estado } \\
\text { nutricional e o risco gestacional. }\end{array}$ & $\begin{array}{l}\text { Os resultados mostraram uma relação significativa entre o } \\
\text { estado nutricional pré-gestacional e gravídico com as síndromes } \\
\text { hipertensivas, reforçando a importância da intervenção } \\
\text { nutricional precoce no pré-natal, a fim de potencializar o } \\
\text { tratamento e o controle dessa morbidade. }\end{array}$ \\
\hline
\end{tabular}

Fonte: Carvalho BL, et al., 2021. tratamento e o controle dessa morbidade. 
Ao analisar os estudos, de acordo com a temática abordada por cada um, pode-se observar que estes em sua maioria têm em comum o enfoque principal dado a importância da identificação dos fatores associado ao desenvolvimento de diabetes mellitus gestacional. Com base nos achados, emergiram as seguintes categorias: Principais fatores de risco relacionados ao desenvolvimento de DMG, Complicações materno fetais em gestantes com DMG, Diagnostico, Tratamento.

\section{Principais fatores de risco relacionados ao desenvolvimento de DMG}

De acordo com Araújo IM, et al. (2020), a diabetes mellitus gestacional (DMG), af eta cerca de $25 \%$ das mulheres grávidas no mundo. Dentro os principais fatores associados ao seu desenvolvimento estão a baixa estatura $(<150 \mathrm{~cm})$, diabetes familiar, idade maior que 25 anos, uso de drogas hiperglicemiantes, obesidade ou grande ganho de peso na gestação, uso de corticoides ou diuréticos, antecedentes obstétricos de morte fetal ou neonatal, macrossomia, malformações, polidrâmnio ou diabetes gestacional.

Segundo Kunzendorff BA, et al. (2017), durante a gestação há uma modificação nos hábitos alimentares, na prática de atividade física, no estado emocional e principalmente mudanças hormonais, fato estes que ocasionam alterações no metabolismo do carboidrato e na necessidade de insulina. Desta forma ocorre a maior procura do feto por glicose, aminoácidos, ácidos graxos e colesterol, o que causa adaptações metabólicas, que se manifestarão na diabetes gestacional.

Desta forma inúmeros estudos identificaram uma maior associação dos hábitos alimentares, obesidade ou grande ganho de peso na gestação a DMG. Estudo realizado por Martins GKF, et al. (2020), no qual a prevalência de DMG foi de 18,5\%, o autor identificou que gestantes que tiveram glicemia em jejum alterada tiveram uma probabilidade $16 \%$ maior de ter DMG. Além disso o autor relata que as doenças mais apresentadas pelas gestantes foram hipertensão arterial (10,66\%) e doença hipertensiva específica da gestação $(2,66 \%)$.

Corrobora com o achado o estudo realizado por Nogueira MDA, et al. (2020), que identificou uma predominância de diabetes de $36 \%$ no DMG e sobrepeso. Com relação à hipertensão, o grupo com DMG e sobrepeso apresentou as maiores taxas de incidência (26,8\% e 3,1\%, respectivamente). O excesso de peso e DMG isolados, ehipertensão, mostraram-se semelhantes ao DMG e o excesso de peso no desenvolvimento do diabetes.

Cysneiros GF, et al. (2020) identificou em seu estudo que cerca de $78,9 \%$ das gestantes estavam com excesso de peso antes da gestação e $80,7 \%$ durante o período gestacional. O ganho ponderal gestacional estava inadequado em $82,5 \%$, estas apresentavam DMG (82,5\%) e apresentava HAS associada (70,2\%). Resultado similar foi evidenciado no estudo de Neris VA, et al. (2021), que avaliou 101 gestantes em seu estudo e identificou que a maioria $(79,2 \%)$ apresentavam excesso de peso prévio e com elevação durante a gestação $(85,1 \%)$.

De acordo com Guerra JVV, et al. (2018), a DMG é uma das intercorrências que mais acometem as gestantes com histórico de sobrepeso pregresso ou que apresentam ganho ponderal inadequado durante a gestação. Em seu estudo o autor identificou que cerca de 14\% das gestantes desenvolveram DMG ao longo da gestação, e 24,5\% iniciaram a gestação com sobrepeso. Observou-se na análise da relação entre o estado nutricional pregresso e o desenvolvimento de DMG que houve associação significativa $(p=0,001)$, desta forma o estudo ressalta que o estado nutricional pregresso esteve fortemente associado à ocorrência de diabetes nesta população.

\section{Complicações materno-fetais em gestantes com DMG}

Segundo Neris VA, et al. (2021), o ganho ponderal excessivo e a obesidade são fatores relacionados ao aumento de complicações materno-fetais em gestantes com DMG, principalmente naquelas que apresentam fatores de risco associados a patologia, como histórico sociodemográfico, clínico e obstétrico

De acordo com Friedrich F, et al. (2019), gestante portadora de DMG não tratada tem maior risco de rotura prematura de membranas, parto pré-termo, feto com apresentação pélvica e feto macrossômico, hipoglicemia e a morte perinatal, bem como maior risco de pré-eclâmpsia. 
No que diz respeito ao feto, este além da macrossomia também tem maior risco para o desenvolvimento de síndrome de angústia respiratória, cardiomiopatia, icterícia, hipoglicemia, hipocalcemia, hipomagnesemia e policitemia com hiperviscosidade sanguínea (MORAIS AM, et al., 2018).

Estudo realizado por Faleiros GQA, et al. (2021), no qual buscou comparar o peso dos recém-nascidos de gestantes com diabéticas gestacional a gestantes metabolicamente normais observou que não houve associação entre o peso materno (peso inicial, peso final, ganho depeso) e peso do recém -nascido, avaliados através da regressão linear. No entanto, diverge como achado, o estudo realizado por Martins GKF, et al. (2020) que observou uma pequena diferença no peso médio levemente superior entre os neonatos de mães com DMG e as mães sem diabetes $(3052,25 \mathrm{~g}$. versus $3011,91 \mathrm{~g}$.).

\section{Diagnostico}

O diagnóstico da DMG, de acordo com a Sociedade Brasileira de Diabetes (SBD) e a Organização Mundial de Saúde (OMS), deve ser realizado por meio do teste de tolerância à glicose (TOTG), que deverá ser realizado entre a 24 e 26 semanas de gestação em todas as grávidas, com sobrecarga de $75 \mathrm{~g}$ de glicose e medidas de glicemia aos 0,1 e 2 horas após.

Considera-se diabetes gestacional as gestantes na qual o teste de tolerância à glicose apresentar valor igual ou superior a 92, 180 e $153 \mathrm{mg} / \mathrm{dL}$ (miligrama decilitro). Nos casos em que o resultado tenha valores iguais ou superiores a 180 e $153 \mathrm{mg} / \mathrm{dl}$, na primeira e segunda hora é de suma importância que a gestante seja encaminhada para o acompanhamento gestacional de risco até o parto (ZUCCOLOTTO DCC, et al., 2019).

\section{Tratamento}

Inúmeros estudos destaca a DMG como um problema de saúde pública, no entanto observa-se que as gestantes têm pouca ou nenhuma informação com relação a DMG. O fato é destacado no estudo realizado por Morais AM, et al. (2018), no qual identificou-se que as grávidas não possuíam conhecimento sobre diabetes, embora 90\% tenham afirmado possuir informação e atualização sobre temas de saúde.

Corrobora com o achado o estudo realizado por Borges MCV, et al. (2017), com 17 gestantes acompanhadas pelo serviço de saúde. O resultado obtido no estudo identificou que $88 \%$ das gestantes desconheciam sobre a DMG e suas implicações para o binômio mãe/filho. $O$ fato se torna preocupante visto a importância do conhecimento das gestantes para melhor adesão ao tratamento da DMG.

De acordo com Araújo IM, et al. (2020), o controle glicêmico rotineiro e o acompanhamento frequente com um profissional de saúde numa maternidade neonatal são fatores importantes para a redução de danos decorrentes da DMG à gestante. Desta forma o enfermeiro deve identificar diagnósticos para elaboração de um plano de cuidados que contribua na prevenção de complicações, através de orientações, acompanhamento e incentivo à manutenção do tratamento (GUERRA JVV et al., 2019).

O plano de cuidados deve priorizar, um melhor controle da nutrição por meio de uma alimentação balanceada, encorajamento à monitoração, a prática de atividades físicas como caminhadas, hidroginástica, o uso correto das medicações, bem como o esclarecimento de toda a enfermidade à paciente e sua família (ARAÚJO IM, et al., 2020).

A terapia nutricional é a primeira opção de escolha pelos profissionais para o tratamento das gestantes com DMG, já a prática de exercício físico leve a moderado só deve ser incentivada na ausência de contraindicações obstétricas. De acordo com estudo de Morais AM, et al. (2018), o tratamento com insulina exógena será indicado quando a dieta e o exercício físico não conseguir normalizar a glicemia da gestante.

O fato é ressaltado no estudo realizado por Martins GKF, et al. (2020), que evidenciou a necessitaram de aplicação de insulina para controlar a glicemia de apenas $23 \%$ das mulheres com DMG, no qual pode-se evidenciar que $77 \%$ das gestantes tiveram bons resultados com tratamento conservador, como dieta e exercícios. Desta forma a prática de exercícios é utilizado como uma alternativa eficaz na terapêutica da DMG, desde intensidade moderada ou leve e com correta indicação pela equipe médica. 
Segundo Faleiros GQA, et al. (2021), fatores de risco como o ganho excessivo de peso, obesidade e hipertensão pode ser tratado com os exercícios físicos e terapia nutricional e com um pré-natal eficiente podese alcançar uma glicemia materna mais próxima da normalidade, fato este que faz diferença no prognóstico materno/fetal e dá a mãe e ao feto uma gestação mais saudável.

\section{CONSIDERAÇÕES FINAIS}

Por meio do presente estudo pode-se analisar na literatura cientifica os principais fatores relacionados ao desenvolvimento da diabetes gestacional, ficando evidente que está é uma da doença que acomete grande parte das gestantes. Houve o relata de uma quantidade significante de gestantes que não tinham conhecimento sobre a DMG, o fato se torna preocupante visto que o conhecimento das gestantes sobre a doença interfere diretamente na adesão ao tratamento. No que diz respeito aos fatores associados ao desenvolvimento da DMG o presente estudo evidencio que a obesidade, o excesso de peso e a péssima nutrição das gestantes estiveram maior relação ao desenvolvimento da DMG, assim como a hipertensão, fato este que torna evidente a importância do acompanhamento das gestantes por uma equipe multiprofissional que contribua para uma gestação mais saudável. O presente estudo mostra-se importante dada a temática abordada que possibilitará aos profissionais da saúde aprimorarem os seus conhecimentos acerca do tema.

\section{REFERÊNCIAS}

1. ARAÚJO IM, et al. Cuidados de enfermagem à pacientes com diabetes mellitus gestacional. Revista Brasileira Interdisciplinar de Saúde, 2020;2(1): 43-8.

2. BATISTA MJ, et al. Diabetes Gestacional: Origem, Prevenção e Riscos. Brazilian Journal of Development, 2020; 7(1): 1981-1995.

3. BORGES MCV et al. O conhecimento das gestantes sobre o diabetesmellitus gestacional em unidade de pré-natal no sul de Minas Gerais. Arch Health Invest, 2017, 6(8): 348-351.

4. CARVALHO BL, et al. Assistência de enfermagem a pacientes com estoma intestinal. Revista Eletrônica Acervo Saúde, 2019;24(64):01-08.

5. CYSNEIROS GF, et al. Estado nutricional e consumo alimentar de gestantes diabéticas atendidas em hospital de referência em Recife-PE. Braz. J. of Develop, 2020;6(7): 463-468.

6. FALEIROS GQA, et al. Diabetes Mellitus Gestacional: o controle glicêmico como elemento de controle de peso fetal.

Revista Eletrônica Acervo Saúde, 2021;13(5):391-398.

7. FRIEDRICH F, et al. Fatores que interferem na adesão ao tratamento da Diabetes Mellitus Gestacional. Revista Saúde e Desenvolvim ento, 2019;13(14):56-62.

8. FERREIRA AF, et al. Diabetes Gestacional: Serão os Atuais Critérios de Diagnóstico Mais Vantajosos?. Revista Científica da Ordem dos Médicos, 2018;31(7-8):416-424.

9. FERNANDES CN, et al. O Diabetes Mellitus Gestacional: Causa e Tratamento. Revista multidisciplinar e de psicologia, 2020;14(49):127-139.

10. GUERRA JVV, et al. Diabetes gestacional e assistência pré-natal no alto risco. Rev enferm UFPE, 2019;13(2): 44954.

11. GUERRA JVV, et al. Diabetes gestacional e estado nutricional materno em um hospital univer sitário de Niterói. J Nurs Health, 2018; 8(1): 188-191.

12. MARTINS GKF, et al. Prevalência e fatores associados ao diabetes mellitus gestacional em um serviço de alta complexidade. Research, Society and Development, 2020;9(8): 739-855.

13. MENICATTI M, et al. Diabetes gestacional: Aspectos fisiopatológicos e tratam ento. Arq. Ciênc. Saúde Unipar, 2017; 10(2): $156-164$.

14. MARIANO TF, et al. A atuacão do enfermeiro no cuidado à gestante com diagnóstico de diabetes gestacional. Glob Acad Nurs, 2021;2(1): 97-105.

15. MORAIS AM, et al. Perfil e conhecimento de gestantes sobre o diabetes mellitus gestacional. Revista Brasileira de Enfermagem, 2018;71(6):899-906.

16. NERIS VA, et al. Ganho ponderal e estado nutricional de mulheres portadoras de diabetes mellitus gestacional. Research, Society and Development, 2021;10(3): 485-494.

17. NOGUEIRA MDA, et al. Associação entre estado nutricional, diabetes gestacional e doenças hipertensivas em gestantes de risco. Braz. J. of Develop, 2020;6(2): 8005-8018.

18. OLIVEIRA ACV, et al. Diabetes Mellitus Gestacional: uma revisão narrativa. Revi sta Eletrônica Acervo Saúde, 2021; 13(5): 189-194.

19. QUEIROZ A, et al. Perfil nutricional e fatores associados em mulheres com diabetes gestacional. Nutricion clinica y dietetica hospitalar, 2018;36(2): 96-102.

20. KUNZENDORFF BA, et al. Influência da diabetes mellitus no período gestacional como fator de risco. Rev Saude Publica, 2017;4(35):221-230.

21. SOUSA AL, et al. Hábitos alimentares saudáveis na prevenção da diabetes gestacional. Revista Eletrônica Acervo Saúde, 2017;20(10):1005-1012.

22. SOUZA W, et al. O acompanhamento multiprofissional da diabetes gestacional na unidade básica de saúde. Rev Inic Cient, 2021;4(2): 676-84.

23. SANTOS WMS, et al. Medidas para proteger o feto de mãe diabética: revisão in tegrativa. Revista Brasileira de Pesquisa em Ciências da Saúde,2019;6(12):35-41.

24. ZUCCOLOTTO DCC, et al. Padrões alimentares de gestantes, excesso de peso materno e diabetes gestacional. Rev Saude Publica, 2019;53(52):378-393. 